\title{
The role of "nothing" in memory for event duration in pigeons
}

\author{
SANTINO C. GAITAN and JOHN T. WIXTED \\ University of Califormia, San Diego, La Jolla, California
}

\begin{abstract}
The research reported herein is designed to test a signal detection account of the choose-short effect, which is the tendency of birds to report (after a long delay) that a short-duration sample was presented, regardless of whether a short or long sample initiated the trial. According to the detection account, the choose-short effect arises because birds learn to selectively search memory for evidence that the long sample appeared on a given trial. This idea is tested, in part, by replacing short-sample trials with nosample trials and showing that performance is unaffected by this manipulation for birds exhibiting a choose-short effect. In addition, when no samples and long samples were associated with the same choice alternative (and the short sample was associated with the other alternative), birds were flexible enough to learn to respond on the basis of the presence versus the absence of the short sample (and, as a result, a choose-long effect was observed).
\end{abstract}

The study of memory for event duration in pigeons has, for the most part, centered on an unusual behavioral phenomenon and an interesting theoretical explanation for it. A typical experiment on memory for event duration involves trials initiated by a sample stimulus that is either short or long in duration (e.g., 2 vs. $10 \mathrm{sec}$ of houselight) and that is later followed by the simultaneous presentation of two choice stimuli (e.g., red and green). A response to one stimulus (e.g., green) is reinforced on trials initiated by the longer sample stimulus, and a response to the other (red) is reinforced on trials initiated by the shorter sample stimulus. The unusual finding is that the forgetting functions following the two samples are reliably asymmetric, in that pigeons tend to choose the alternative associated with the short sample following a lengthy retention interval, regardless of which sample was actually presented. As a result, performance following the long sample decreases rapidly to well below $50 \%$ correct as the retention interval increases, whereas performance following the short sample remains accurate at well above 50\% correct (Fetterman, 1995; Spetch, 1987; Spetch \& Wilkie, 1982, 1983). This increasing bias to choose the alternative associated with the short sample as the retention interval increases is called the choose-short effect.

\section{SUBJECTIVE SHORTENING}

Why does this procedure yield asymmetric forgetting functions? The dominant theoretical interpretation of

These experiments were conducted while the primary author was an NSF Predoctoral Fellow, and the research was supported by NIMH Grant MH55648. Correspondence concerning this article should be addressed to J. T. Wixted, Department of Psychology, 0109, University of California, San Diego, La Jolla, CA 92093-0109 (e-mail: jwixted@ ucsd.edu). this pattern of results is that the remembered duration of an event decreases with the passage of time. Thus, immediately after it is presented, a long-duration sample will be accurately represented (leading to accurate performance). As the retention interval increases, however, that representation will continuously decrease. Eventually, when the retention interval is long enough, the longduration sample will actually be remembered as having been short in duration. At that point, the pigeon selects the choice alternative associated with the short sample, even though the trial actually began with the long sample. Note that the opposite bias (i.e., a bias to select the long choice alternative on short-sample trials) would not be expected, because any decrease in the remembered duration of the short sample as the retention interval increases would not create a tendency to choose the long choice alternative. Instead, the bird would presumably continue to choose the short alternative. Hence, the asymmetric pattern in favor of the short sample. The theoretical decrease in the representation of event duration with the passage of time is known as subjective shortening (Spetch \& Wilkie, $1982,1983)$. This account has been widely investigated and has been shown to be consistent with a wide array of findings based on this procedure and variants of it.

\section{MEMORY FOR ASYMMETRIC EVENTS}

A different and less widely investigated theoretical interpretation of the asymmetric forgetting functions produced by this procedure is based on the idea that memory for "something versus nothing" plays a prominent role, a point initially made by Kraemer, Mazmanian, and Roberts (1985). Important information about how this might play a role in memory for event duration is provided by studies in which memory for the presence versus the absence of an event (i.e., memory for something vs. nothing) has specifically been investigated. In a typical 
experiment of this kind, sample and no-sample trials are randomly intermixed, and the pigeon's task is to report whether or not a sample appeared. On sample trials, the end of the intertrial interval (ITI) is followed by the presentation of a sample (e.g., the brief illumination of a keylight or the houselight) and then by the retention interval. On no-sample trials, by contrast, the end of the ITI is followed immediately by the onset of the nominal retention interval (i.e., no sample is presented). Following the retention interval, two choice stimuli are presented. A response to one choice alternative is correct on sample trials, and a response to the other is correct on no-sample trials.

Grant (1991) and Wixted (1993) both used this procedure to study memory for the presence or absence of keylights. Several other researchers have used a similar procedure to test memory for the presence or absence of food (Colwill, 1984; Sherburne \& Zentall, 1993; Wilson \& Boakes, 1985). In the latter studies, responding to one choice stimulus was reinforced following a brief presentation of a food sample, whereas responding to the other choice stimulus was reinforced if food had not been presented at the beginning of the trial. The pattern of results emerging from all of these studies is that performance on the no-sample (or no-food) trials is quite accurate and does not change much as a function of retention interval, whereas performance on sample trials is nearly perfect at short retention intervals and drops to well below $50 \%$ correct at longer retention intervals.

Performance on a no-sample trial is theoretically determined by the absence of memory for a sample, and that memory is absent whether the retention interval is short or long (Grant, 1991; Wixted, 1993). Thus, because there is nothing to forget on these trials, the retention function remains flat. On sample trials, by contrast, memory for the sample is strong following a short retention interval, which allows the pigeon to make the correct choice on that basis. After a long retention interval, however, memory for the sample will have faded away. At that point, the sample trial is psychologically equivalent to a no-sample trial, and pigeons have learned that the other choice alternative is correct when memory for the sample is absent. Thus, they choose the wrong alternative more than $50 \%$ of the time.

What is the relationship, if any, between the asymmetric forgetting functions observed in a presence versus absence procedure and those observed in an event duration procedure? Different theoretical accounts are usually offered to explain the similar performance produced by the two tasks, but a common mechanism could be involved. Indeed, one straightforward (although not necessarily correct) assumption leads to the prediction that asymmetric forgetting functions should be observed whenever one sample differs in salience from the other. Whenever that situation exists, pigeons may be inclined to transform the discrimination task into a detection task based on the presence or absence of the more salient sample (with memory for the less salient sample playing no role in the choice decision).
In an event duration procedure, the 10 -sec sample is presumably a more salient event than the 2 -sec sample. Thus, the pigeon's decision strategy may not be based on the discrimination between the two samples ("Did the 10 -sec or the 2-sec sample occur?") but may, instead, be based on the detection of the 10-sec sample ("Did the 10sec sample occur or not?"). One way to illustrate the difference between a discrimination strategy and a detection strategy is to assume that the individual choice stimuli presented at the end of a trial act as sample-specific retrieval cues. A bird relying on a discrimination strategy might examine the choice stimulus associated with the 2sec sample (e.g., red) and attempt to retrieve a memory of that event. Next, the bird might examine the choice stimulus associated with the 10-sec sample (e.g., green) and attempt to retrieve a memory of that event. The bird's choice would be dictated by the sample duration that yields the strongest evidence of having occurred on that trial.

A bird using a detection strategy would also examine the choice stimulus associated with the 10 -sec sample (e.g., green) and attempt to retrieve a memory of that event. If the strength of the retrieved memory for the long sample exceeded a decision criterion, the green choice alternative would be selected. If not, red would be selected (see Wixted, 1993). According to this idea, retrieval of memory for the 2-sec sample is not even attempted. This account is similar to a default response model, which assumes that birds select the choice alternative associated with the 2-sec sample by default (Grant \& Spetch, 1994).

If pigeons did adopt a detection strategy like this, a choose-short effect would be expected even if remembered duration did not decrease with the passage of time. According to the detection model, the birds in an event duration task learn to choose, say, green in the presence of memory for the 10-sec sample and to choose red in the absence of memory for that sample (i.e., on 2-sec-sample trials). How should an increasing retention interval affect performance under these conditions? On every trial, the bird is assumed to attempt retrieval of memory for the 10-sec sample. On 10-sec sample trials, performance following a short retention interval would be accurate because memory for the 10 -sec-sample would be strong at that point. As the retention interval increased and memory for the 10-sec sample weakened, however, performance would decline. When memory for the 10 -sec sample faded completely, the bird would make the choice it has learned to make in the absence of memory for that sample (i.e., it would select red, the alternative associated with 2 -sec samples). On a 2-sec-sample trial, by contrast, memory for the 10 -sec sample is equally absent whether the retention interval is very short or very long. Because the absence of a memory trace does not decay, the forgetting function should be flat. Thus, this account predicts the widely observed choose-short effect.

The three experiments described herein tested the viability of this account. The first experiment was designed to test the idea that the 2-sec-sample trials are expendable because they have no influence on the choice decision anyway. Some evidence in support of this idea has already 
been provided by studies that introduced no-sample probe trials in an otherwise standard short versus long event duration procedure. The results of these studies reliably show that pigeons have a bias to select the choice stimulus associated with the short sample on these nosample probe trials (e.g., Fetterman \& MacEwen, 1989; Spetch \& Wilkie, 1983). The present experiment takes this one step further by testing how important the actual presentation of the short sample is. This was investigated by training pigeons on a standard $2-\mathrm{sec}$ versus $10-\mathrm{sec}$ procedure to stability, followed by five sessions in which the 2-sec-sample trials were replaced by no-sample trials (but all else was the same), followed by five sessions in which the no-sample trials were changed back to 2sec-sample trials. If memory for the 2-sec sample exerts no influence over the bird's choice behavior (as the detection analysis assumes), this manipulation should have no measurable effect on performance.

The second experiment was similar to the first, except that training involved 2- and 10-sec samples (as before), as well as explicitly programmed no-sample trials. On these trials, no sample was presented following the ITI, and the choice stimuli were presented, as usual, following the retention interval. The correct choice on these nosample trials was the same as that on 2-sec-sample trials (e.g., red), whereas the other choice was correct on 10-secsample trials (e.g., green). This procedure establishes a situation in which responding on the basis of the presence or absence of the 10-sec sample is an efficient strategy. That is, instead of applying three rules (if $2 \mathrm{sec}$, choose red; if no sample, choose red; and if $10 \mathrm{sec}$, choose green), the bird could rely on two (if $10 \mathrm{sec}$, choose green; if not, choose red). If pigeons did adopt such a strategy, their performance on 2-sec-sample trials and on no-sample trials should be accurate (because the birds have learned which choice is correct in the absence of memory for the 10 -sec sample) and should be unaffected by the size of the retention interval (because the absence of a trace does not decay). Performance following the 10 -sec sample, by contrast, should be accurate at first but should then decline to below $50 \%$ correct as the retention interval increases (because the eventual absence of memory for the 10 -sec sample induces the bird to choose the wrong alternative).

The third experiment tested whether pigeons, who may usually respond on the basis of the presence or absence of the 10-sec sample, can be induced to respond on the basis of the presence or absence of the 2-sec sample instead. To accomplish this, the procedure used in Experiment 2 was modified in such a way that the correct choice on the no-sample trials was now the same as that on 10sec sample trials (e.g., green). Under these conditions, the pigeon can no longer respond efficiently on the basis of the presence versus the absence of the $10-\mathrm{sec}$ sample because, in the absence of memory for the 10-sec samples, the correct choice is sometimes green (e.g., when no sample is presented) and sometimes red (e.g., when the 2-sec sample is presented). On the other hand, the pigeon could respond efficiently on the basis of the presence versus the absence of the 2-sec sample (i.e., if the 2-sec sample occurred, choose red; otherwise, choose green).

What should happen if pigeons did respond in this way? Because memory for the 10 -sec sample would not be involved in the choice decision, performance on those trials should remain accurate and flat as the retention interval increases. Indeed, performance on 10-sec trials should not differ from performance on no-sample trials in any way (because performance on both kinds of trial is determined by the absence of memory for the 2-sec sample). On 2-sec-sample trials, by contrast, performance should be accurate when the retention interval is short and then decline to less than $50 \%$ correct as the retention interval increases (as the memory of the 2-sec sample fades away to nothing). In other words, a strong choose-long bias should be observed, which is exactly the opposite of what is usually observed.

It should be emphasized at the outset that the experiments reported here do not provide a differential test of the signal detection versus subjective shortening accounts. Rather, the experiments were primarily designed to test the viability of the detection account. Still, because the subjective shortening model has effectively guided thinking about memory for event duration for some time, we do consider what that account might have to say about the results of each experiment.

\section{EXPERIMENT 1}

In the first experiment, we investigated the effect of replacing 2-sec-sample trials with no-sample trials after pigeons had acquired the $2-\mathrm{sec}$ versus $10-\mathrm{sec}$ discrimination. If, at some point during the course of training, pigeons learn to respond on the basis of the presence or $a b-$ sence of the 10-sec sample, replacing the 2-sec-sample trials with no-sample trials for five sessions should have little or no effect on performance. Subsequently reinstating the 2-sec-sample trials should be equally inconsequential.

\section{Method}

Subjects. Four White Carneau and four Colombia Livia pigeons with previous experience on symbolic delayed matching-to-sample (DMTS) tasks were maintained at approximately $80 \%$ of their freefeeding weights. The birds were weighed before each experimental session and afterward were fed an appropriate amount of mixed birdseed and pigeon chow. Water and grit were available ad lib in the home cages.

Apparatus. The two experimental chambers used were $35 \mathrm{~cm}$ long, $32 \mathrm{~cm}$ wide, and $34 \mathrm{~cm}$ high. Each chamber was constructed of Plexiglas, except for one wall, which was metal. Three Plexiglas response windows, each $6.5 \mathrm{~cm}$ high and $4.4 \mathrm{~cm}$ wide, were mounted in the metallic wall of the chambers $20 \mathrm{~cm}$ above the chamber floor (in all three experiments, choice stimuli appeared only on the outside two keys-never on the middle). A VGA color monitor was directly visible through the response key windows and displayed the choice stimuli. The distance from the response surface to the mon- 


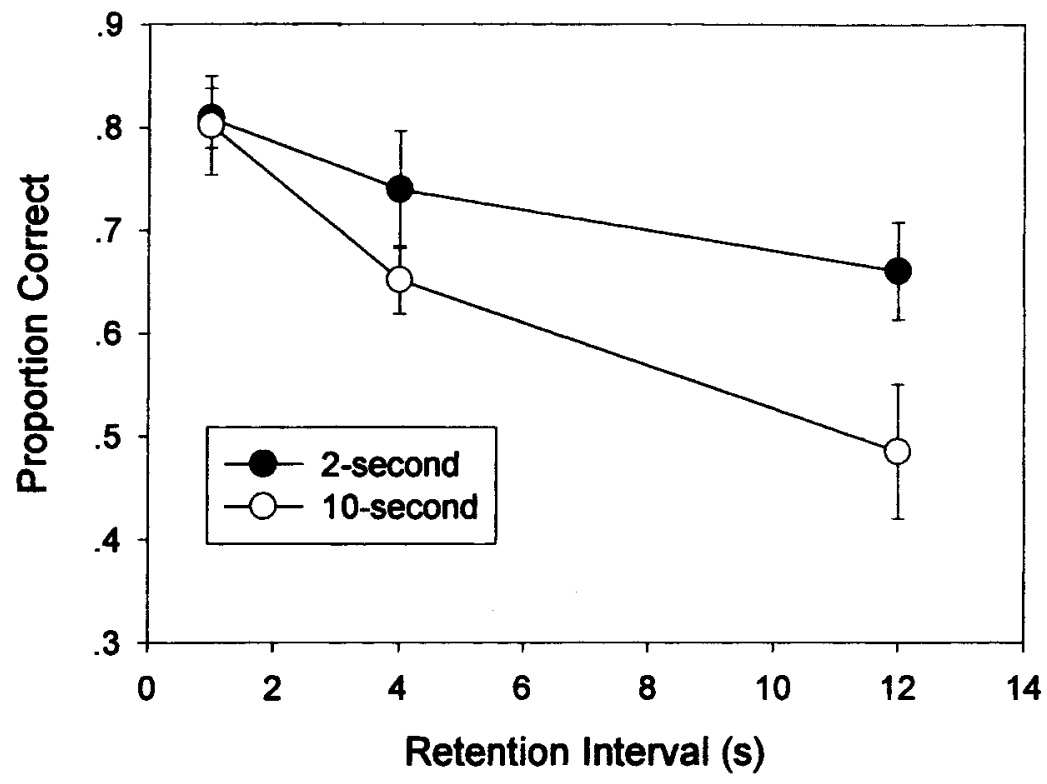

Figure 1. Proportion of correct responses as a function of retention interval for the short (2-sec) and long (10-sec) samples, averaged over the 8 birds from Experiment 1.

itor surface was approximately $5 \mathrm{~cm}$. The sample stimulus consisted of a 1.2-W overhead houselight located on the ceiling $8 \mathrm{~cm}$ from the rear wall. The houselight varied only in the duration of illumination across the various stimuli. One choice stimulus consisted of two vertically stacked $2.5-\mathrm{cm}$ red circles, whereas the other choice stimulus consisted of five $1.5-\mathrm{cm}$ green squares arranged in a checkerboard pattern. Both choice stimuli were presented on a 6cm-square white background. Pecks were recorded via a microswitch located at the base of each hinged window. A pellet tray located below the center window and $4 \mathrm{~cm}$ above the chamber floor collected Noyes $45-\mathrm{mg}$ food pellets dispensed by a Gerbrands precision pellet dispenser. The chambers were not illuminated during the experimental sessions (except by the sample stimuli), and an exhaust fan masked extraneous noise. Each chamber and respective VGA monitor were enclosed in a sound- and light-attenuating chamber. The experiment was controlled by an IBM-compatible computer that also recorded the data. A closed circuit video camera and microphone inside each chamber allowed visual and aural monitoring of the birds during test sessions.

Procedure. All 8 birds had previous experience on the delayed discrimination procedure prior to beginning this experiment. In the training phase of the present experiment, the houselight was off, except during presentation of the sample stimuli. Following a 15-sec ITI, a sample consisting of either a 2-sec or a 10-sec illumination of the houselight was presented. This was followed by a retention interval of 1,4 , or $12 \mathrm{sec}$, which varied randomly from trial to trial. Following the retention interval, red and green choice stimuli were presented on the two side keys. A single response to one choice stimulus was correct on 2-sec-sample trials, and response to the other was correct on 10-sec-sample trials (colors were counterbalanced across birds). Correct responses were reinforced with two food pellets accompanied by a $5-\mathrm{sec}$ hopper light. Any response to the choice keys immediately extinguished the choice stimuli and resulted either in reinforcement or, if incorrect, in initiation of the ITI.

The pigeons were run under these conditions for 45 sessions prior to beginning Experiment 1. During these 45 sessions, the bal- ance of 2-sec- and 10-sec-sample trials was varied over blocks of 15 sessions. In one block, $50 \%$ of the trials were initiated by the 2 -sec sample, and $50 \%$ by the 10 -sec sample. In another block, $80 \%$ of the trials were initiated by the $2-\mathrm{sec}$ sample, and $20 \%$ by the 10 -sec sample, and in a third block, $20 \%$ of the trials were initiated by the 2 -sec sample and $80 \%$ by the 10 -sec sample. The data collected during these sessions were part of another experiment and are not reported here.

After completing those 45 sessions, all the pigeons were placed back on the procedure in which $50 \%$ of the trials were initiated by the 2 -sec sample and $50 \%$ by the 10 -sec sample for another block of 15 sessions. The last 5 sessions of this block constituted Phase 1 of Experiment 1. Phase 2 consisted of the next 5 sessions and was procedurally identical to Phase 1 , except that 2 -sec-sample trials were replaced by $2 \mathrm{sec}$ of continued darkness. That is, the end of the ITI blended seamlessly into the beginning of the retention interval. These trials are henceforth referred to as no-sample trials. If the red choice stimulus had been correct for the 2-sec-sample trials in Phase 1, red was the correct choice on no-sample trials during Phase 2. During Phases 1 and 2, 10-sec-sample trials were identical in every respect. Finally, in Phase 3, no-sample trials were replaced once again by 2 -sec samples (i.e., Phase 1 and Phase 3 were procedurally identical).

\section{Results and Discussion}

The data from each subject were averaged over the five sessions of each phase. Figure 1 shows performance in Phase 1 on the $2-\mathrm{sec}$ and $10-\mathrm{sec}$ trials as a function of retention interval (averaged over subjects). It is evident from the figure that a choose-short bias was obtained. That is, the forgetting function following the 10-sec sample is steeper than that following the 2-sec sample. An analysis of variance (ANOVA) performed on these data revealed a main effect of retention interval $\left[F(2,14)=26.97, M S_{\mathrm{e}}=\right.$ 

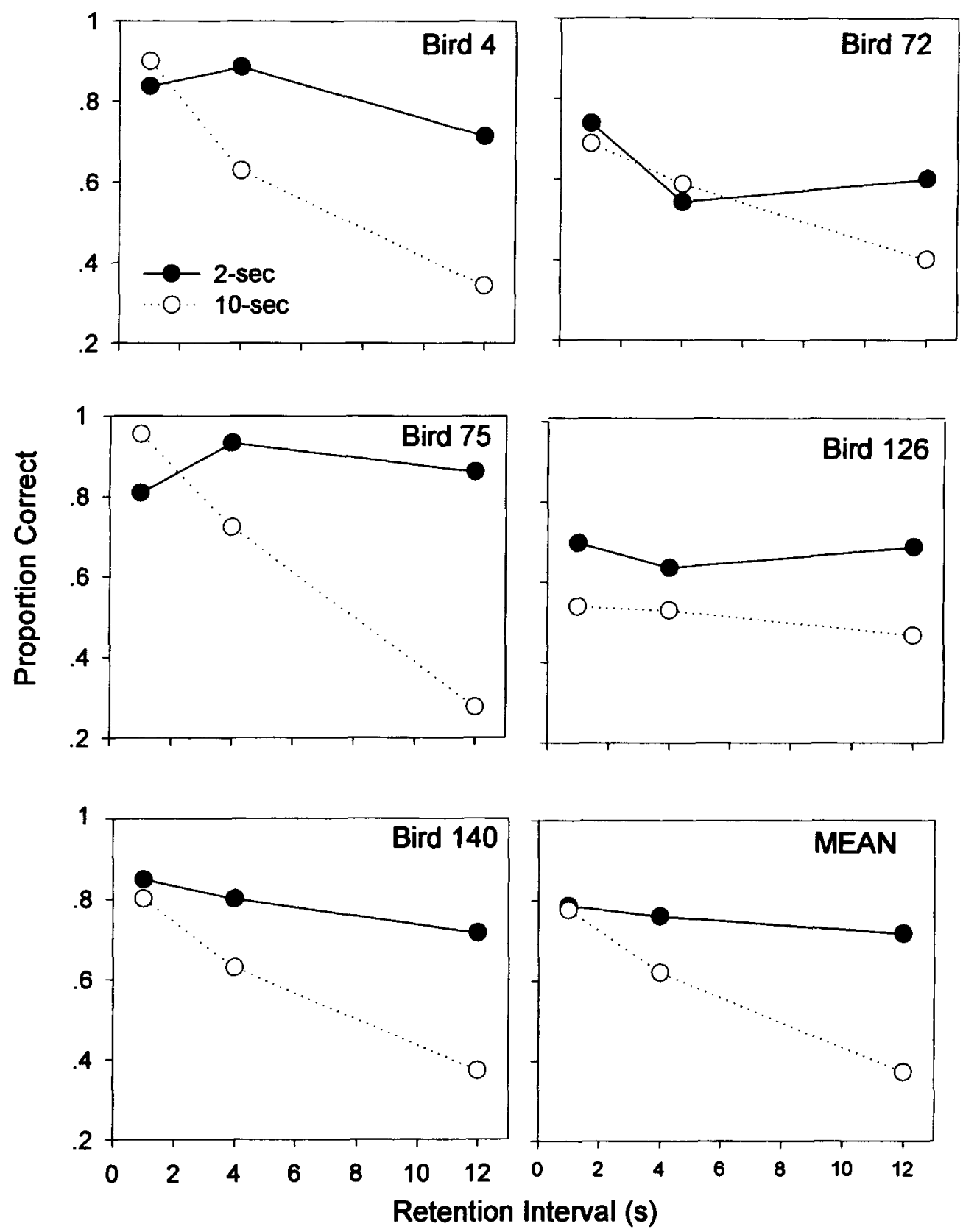

Figure 2. Proportion of correct responses as a function of retention interval on the 2-sec-sample and 10sec-sample trials for the 5 birds in Experiment 1 that exhibited a choose-short effect. The lower right panel shows the mean for these 5 birds.

$0.008, p<.05]$ and a marginally significant effect of sample duration $\left[F(1,7)=5.05, M S_{\mathrm{e}}=0.019, p=.059\right]$, but the interaction was not significant.

An analysis of the individual subject data revealed that the choose-short pattern evident in Figure 1 was not representative of all 8 subjects. Indeed, as is shown in Figures 2 and 3, two distinct patterns of response to the retention intervals were found. Five of the birds (Figure 2) showed asymmetric forgetting functions to varying degrees, so that they were more likely to respond correctly on short trials than on long trials at the longest retention interval. Furthermore, for all 5 of these birds, accuracy on 10 -sec-sample trials was below $50 \%$ correct at the longest retention interval (although only slightly so for Bird 126). The average of these 5 birds (also shown in Figure 2) exhibits a pronounced choose-short effect. If this chooseshort effect occurs because these birds have adopted a detection strategy based on the presence or absence of the 10 -sec sample, their behavior should not be affected when the 2-sec samples are replaced by no samples in Phase 2. 

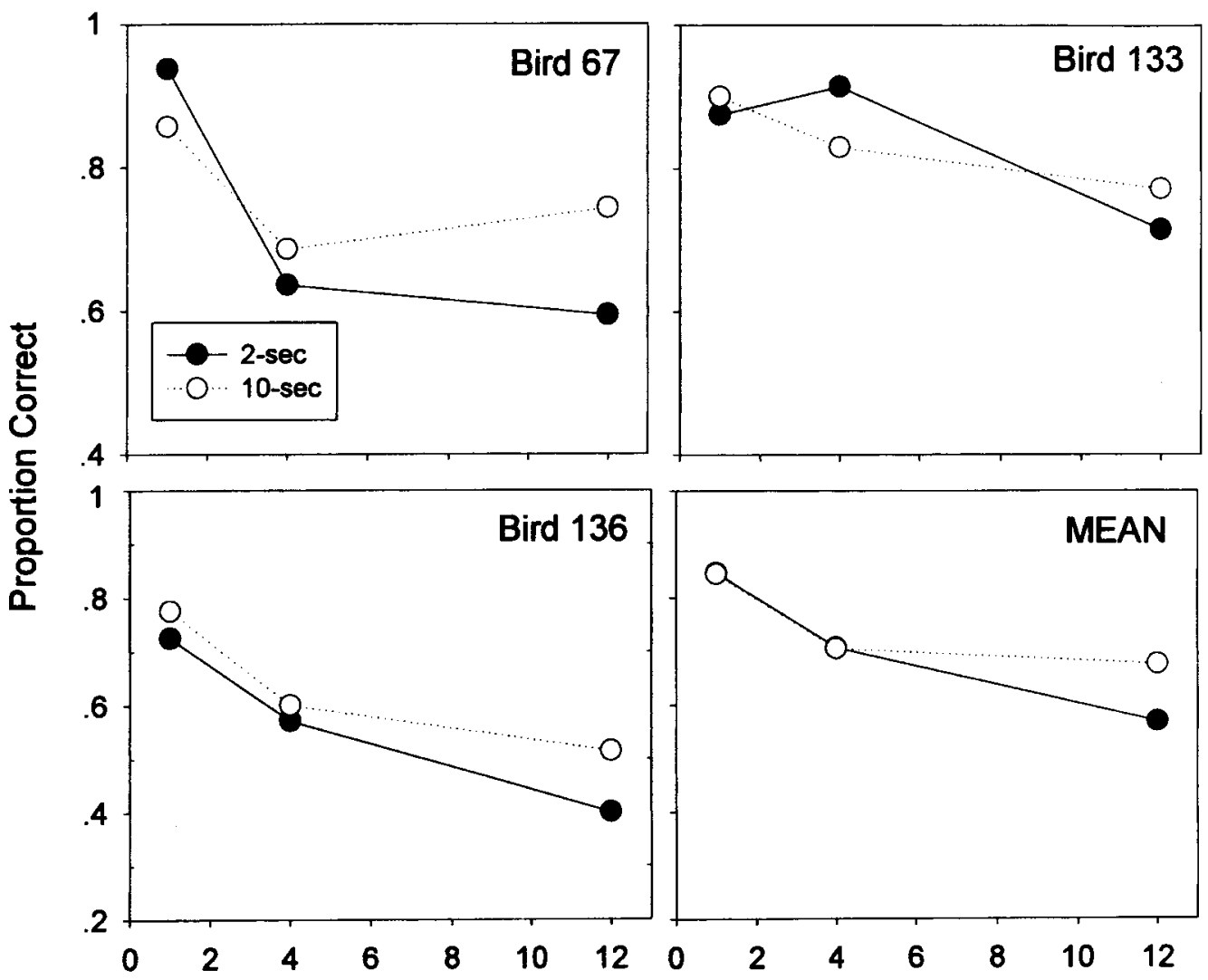

Retention Interval (s)

Figure 3. Proportion of correct responses as a function of retention interval on the 2-sec-sample and 10-secsample trials for the 3 birds in Experiment 1 that did not exhibit a choose-short effect. The lower right panel shows the mean for these 3 birds.

The remaining 3 birds (Figure 3) showed no evidence of asymmetric forgetting. For all 3 birds, performance on short trials was actually slightly worse than performance on long trials at the longest retention interval, and their performance on the long trials never fell below $50 \%$ correct. Thus, for these 3 birds, there is no hint of a chooseshort effect. These birds had experienced more than 60 sessions on a 2 -sec versus 10-sec discrimination procedure, so the pattern reflected in Figures 2 and 3 reflects stable responding. The fact that these 3 birds exhibited a clear forgetting function following the 2-sec samples means that they did not adopt a detection strategy. Rather, they must have relied on a discrimination strategy in which they responded on the basis of whether they remembered the sample as having been 10 or $2 \mathrm{sec}$ in duration. That being the case, the behavior of these 3 birds, unlike that of the other 5 birds, should be affected by the substitution of no-sample trials for 2-sec-sample trials in Phase 2. For this reason, subsequent analyses treat these two groups (namely, the 5 birds who showed evidence of a choose-short effect and the 3 who did not) separately.

The upper panel of Figure 4 shows the main results of Experiment 1 for the 5 birds who showed evidence of a choose-short effect in Phase 1. Because the data from Phases 1 and 3 ( 2 vs. $10 \mathrm{sec}$ ) were very similar and did not differ significantly, those results were averaged together and plotted along with the results from Phase 2 (no sample vs. 10 -sec sample). What is clear from Figure 4 is that replacing the 2-sec-sample trials with no-sample trials for these 5 birds had little or no effect on their performance. Of particular interest is performance on no-sample trials relative to the 2 -sec-sample trials. An ANOVA performed on these data yielded no significant effects. The relatively small error bars indicate that power to detect any effect that might have occurred was reasonably high. Based on the obtained error variance, power to detect a true $10 \%$ difference in performance (averaged across the three retention intervals) was .96 . Of course, smaller differences might not have been detected. Power to detect a true $5 \%$ difference, for example, was .48 . Thus, a true difference that small would be missed about $50 \%$ of the time.

A similar analysis was performed on the data from the 10 -sec-sample trials across the two phases of the experiment. An ANOVA performed on these data revealed a main effect of retention interval $\left[F(2,8)=24.97, M S_{\mathrm{e}}=\right.$ $0.016, p<.05]$, but no other effects were significant. 

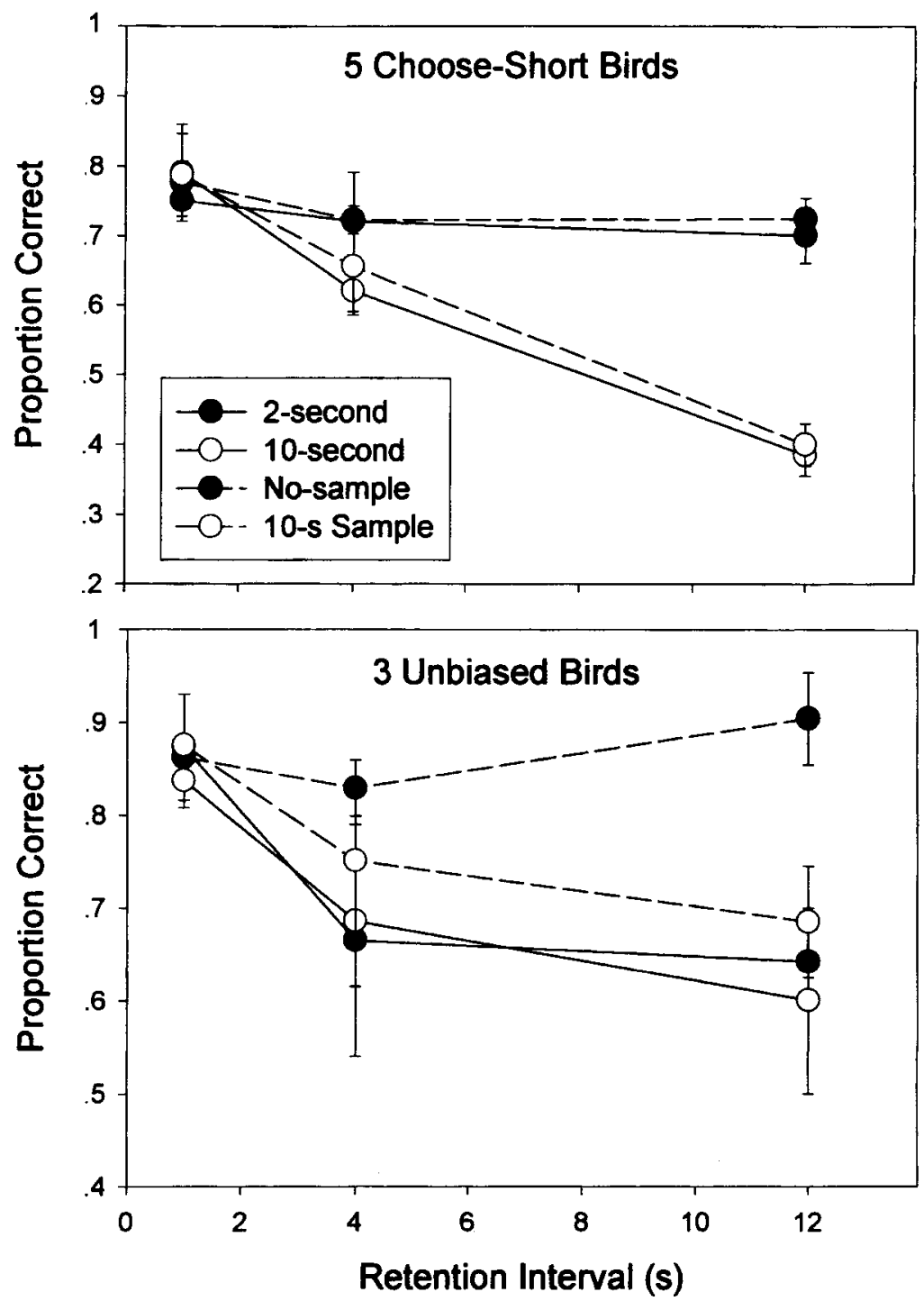

Figure 4. Upper panel: proportion of correct responses as a function of retention interval on 2-sec- versus 10-sec-sample trials (averaged across Phases 1 and 3 ) and on no-sample versus 10-sec-sample trials (Phase 2) for the 5 birds in Experiment 1 that exhibited a choose-short effect. Lower panel: proportion of correct responses as a function of retention interval on 2-sec- versus 10-sec-sample trials (averaged across Phases 1 and 3) and on no-sample versus 10-sec-sample trials (Phase 2) for the 3 birds in Experiment 1 that did not exhibit a choose-short effect.

Again, the relatively small error bars indicate that power to detect any effect that might have occurred was reasonably high. Using the obtained error variance as an estimate of the true error variance, power to detect a true $10 \%$ difference in performance (averaged across retention interval) was .89. Power to detect a true $5 \%$ difference was .36 . Thus, a true difference that small would be missed about two thirds of the time.

The lower panel of Figure 4 shows the comparable results for the 3 birds that showed no evidence of a chooseshort effect in Phase 1. Whereas no forgetting function asymmetry is apparent for the data averaged over Phases
1 and 3, a clear asymmetry is apparent in Phase 2. That is, performance on no-sample trials was unaffected by the size of the retention interval (it actually improved slightly), whereas performance on the 10-sec-sample trials declined as the retention interval increased (the expected pattern on a sample/no-sample task).

The results of Experiment 1 show that for the birds showing evidence of a choose-short effect, replacing 2sec-sample trials with no-sample trials had virtually no effect on their performance. This is the expected result if one assumes that these birds had learned to respond on the basis of the presence versus the absence of the 10 -sec 
sample. If memory for the 2-sec samples was having no influence on choice behavior anyway, eliminating those samples from the procedure should not affect performance.

That pigeons might adopt a detection strategy is understandable, given that the samples in an event duration procedure presumably differ in salience. Because one sample is more salient, the birds may find it easier to respond on the basis of its presence or absence in much the same way as that in which they respond on the basis of the presence versus the absence of food when samples consist of food or no food. In both cases, memory for the less salient event may be disregarded in favor of the more salient event.

Note that there is no procedural requirement that birds transform the 2 -sec versus the 10 -sec discrimination into a 10 -sec versus not-10-sec detection task. Although 5 birds may have done precisely that, the behavior of the other 3 birds in this experiment suggests that they did not. The fact that they exhibited a clear forgetting function following the 2-sec samples in Phase 1 proves that memory for those samples influenced choice performance in a way that decreased as memory for the short sample faded. As might be expected, their behavior was noticeably affected when the 2 -sec-sample trials were replaced by no-sample trials. That is, although no forgetting function asymmetry was apparent in Phase 1 for these birds, the usual asymmetry emerged in Phase 2 (during which the pigeon's job was to report whether or not a $10-\mathrm{sec}$ sample had occurred). Somewhat surprisingly, the asymmetry emerged quite quickly, and the 3 birds responded more accurately in Phase 2 than they did in Phase 1, even though Phase 2 was in effect for only five sessions. Why these birds were so efficient in Phase 2 is not clear. One possible explanation is that these birds had, earlier in training, learned to rely on a detection strategy and easily reverted to it when the procedure was changed to require the detection of a houselight in Phase 2.

The results of Experiment 1 are consistent with the idea that some birds relied on a detection strategy in all three phases, but they are also compatible with a subjectiveshortening interpretation. According to the latter account, the 5 birds that exhibited a choose-short effect did so because they relied on analogical coding of the two samples and because the subjective duration of the long sample decreased with the passage of time in Phases 1 and 3 and perhaps in Phase 2 as well. The 3 birds that did not exhibit a choose-short effect presumably relied on nonanalogical coding, perhaps because varied retention intervals were used throughout training (see Grant \& Kelly, 1998).

The subjective-shortening model does not predict that replacing the 2 -sec-sample trials with no-sample trials will have absolutely no effect on performance (the main result of Experiment 1), but that finding is not necessarily incompatible with the theory either. Conceivably, an absent sample is considered by the bird to be a 0 -sec sample. If so, a 2-sec sample and a 0-sec sample may be sufficiently similar that any difference in performance they support might be too small to detect. This solution may be logically problematic, since a nonevent cannot really be assigned a duration (e.g., a no-sample trial does not actually involve an event that lasted for $0 \mathrm{sec}$ ). Still, perhaps the birds are inclined to treat a nonevent as the end of a temporal continuum, in which case the difference between a 2 -sec event and a $0-\mathrm{sec}$ nonevent might be negligible from the bird's point of view.

An alternative and, perhaps, more likely possibility from the point of view of subjective-shortening theory is that the birds responded on the basis of analogical encoding of 2- and 10-sec samples during Phases 1 and 3 (as usual) but adopted a different strategy during Phase 2 . More specifically, when the procedure involved only one sample (as in Phase 2), the birds may have adopted the detection strategy discussed above, or they may have adopted the closely related default response strategy. According to the default response account, birds choose the alternative associated with no sample by default and choose the other alternative only when memory for the sample is present (memory that is only available following a short retention interval). This is a widely held view of performance on sample/no-sample procedures, and it predicts the asymmetry that is typically observed (Grant, 1991). Although the default response account is not the same as the detection account offered here, the two are sufficiently similar that a discussion of their important differences will be deferred to the General Discussion section.

If one did assume that the birds adopted a default response (or detection) strategy selectively in Phase 2, one would also have to assume that the similarity in performance across the three phases of Experiment 1 is purely coincidental. By contrast, we assume that the similarity arises from the fact that a detection strategy is in effect in all three phases of the experiment (not just in Phase 2).

\section{EXPERIMENT 2}

To further test the idea that pigeons are capable of responding on the basis of a detection strategy, Experiment 2 included no-sample trials throughout the course of training. A response to one choice alternative (e.g., green) was correct on 10-sec-sample trials, and a response to the other (e.g., red) was correct on 2-sec-sample trials and on no-sample trials. With such an arrangement, the pigeons could respond on the basis of three separate rules: if 2-sec, choose red; if no sample, choose red; and if $10 \mathrm{sec}$, choose green. If the birds did respond on that basis, they would not necessarily show equivalent performances on the 2-sec-sample and the no-sample trials, and performance on the 2-sec-sample trials might deteriorate with increasing retention interval (as memory for that sample decayed). Although the birds might respond on the basis of three decision rules in this way, a more efficient retrieval strategy would be to respond on the basis of only two-namely, if $10 \mathrm{sec}$, choose green; otherwise, choose 
Table 1

Assignment of Sample Stimuli (0, 2, and $10 \mathrm{sec})$ to Correct Choice Stimuli in Experiments 2 and 3

\begin{tabular}{|c|c|c|c|c|}
\hline \multirow[b]{2}{*}{ Pigeon } & \multicolumn{2}{|c|}{ Experiment 2} & \multicolumn{2}{|c|}{ Experiment 3} \\
\hline & Red & Green & Red & Green \\
\hline 15 & 10 & 0,2 & 0,10 & 2 \\
\hline 26 & 10 & 0,2 & 0,10 & 2 \\
\hline 27 & 0,2 & 10 & 2 & 0,10 \\
\hline 44 & 0,2 & 10 & 2 & 0,10 \\
\hline
\end{tabular}

red. If the birds did adopt this detection strategy, performance on 2-sec-sample and no-sample trials should be equivalent, and neither should show any effect of retention interval.

Experiment 2 also differed from Experiment 1 in that baseline training sessions always involved a retention interval of $1 \mathrm{sec}$ (rather than using multiple retention intervals during baseline training). In Experiment 2, multiple retention intervals were introduced during occasional probe sessions.

\section{Method}

Subjects. Four White Carneau pigeons, distinct from those used in Experiment 1 and with previous experience on DMTS tasks, were maintained at approximately $80 \%$ of their free-feeding weights. The birds were weighed before each experimental session and afterward were fed an appropriate amount of mixed birdseed and pigeon chow. Water and grit were available ad lib in the home cages.

Apparatus. The same experimental apparatus as that used in Experiment 1 was used again here, except that only one of the two chambers was used.

Procedure. Experiment 2 was actually run after Experiment 3 (which involved the same set of birds), but we present the results of this experiment first because it follows more naturally from Experiment 1 . In initial training for this experiment, each daily session consisted of 60 trials consisting of fifteen 2 -sec-sample trials, thirty 10 -sec-sample trials, and 15 no-sample trials. The 2 -sec and $10-\mathrm{sec}$ samples again consisted of houselight presentations. On no-sample trials, the transition from the dark ITI to a dark retention interval was seamless. Red and green choice stimuli were presented after a $1-\mathrm{sec}$ delay following termination of the sample stimulus (these were the same as those detailed in Experiment 1). A peck to one keylight (green for 2 birds, red for the other 2) was reinforced with two food pellets and a 5-sec hopper light following 2-sec samples and on no-sample trials. A peck to the other choice key was similarly reinforced following presentation of the $10-\mathrm{sec}$ stimulus. Table 1 presents the assignment of samples $(0,2$, or $10 \mathrm{sec})$ to choice stimuli (red and green) for both Experiments 2 and 3 . The only procedural difference between the two experiments concerned the assignment of the correct choice on no-sample $(0-\mathrm{sec})$ trials.

Termination of the hopper light or an incorrect choice initiated a dark ITI of $15 \mathrm{sec}$. Order of stimulus presentation was determined randomly at the beginning of each session, although during initial training, a correction procedure was used, so that a trial was repeated until a correct response occurred. The position of the choice stimuli, left or right key, was determined randomly.

For 3 of the birds, performance remained well above chance on the 2-sec and 10-sec trials when the mapping of the 0-sec sample was switched following Experiment 3 . As might be expected, however, performance on no-sample $(0-\mathrm{sec})$ trials was well below chance for a number of sessions. For Bird 44 , however, overall performance was disrupted and did not reliably exceed chance on all three trial types simultaneously for nearly 30 sessions. After a rea- sonable performance level had been attained for each bird, the correction procedure was discontinued.

Once performance without the correction procedure reached stability (defined as five consecutive sessions above $75 \%$ correct overall), probe sessions were introduced. The probe session differed from the baseline sessions only in the size of the retention intervals that followed the sample stimuli. Whereas baseline sessions used a retention interval of $1 \mathrm{sec}$ on every trial, probe sessions involved retention intervals of $1,2,4$, and $12 \mathrm{sec}$ following each sample. The retention intervals varied within session, were equally likely, and were randomly selected. A total of four probe sessions was run for each bird. Each probe session was followed by two sessions of baseline training, in which all the retention intervals were again $1 \mathrm{sec}$ long.

\section{Results and Discussion}

Figure 5 presents the main results of Experiment 2. The figure shows the performance of each bird on 2-sec, 10-sec, and no-sample trials averaged over the four probe sessions. The bottom panel shows performance averaged over the 4 birds. All 4 birds again demonstrated a clear asymmetry between accuracy following the 2 -sec samples and that following the 10 -sec samples. Accuracy following the 2-sec samples remained high as the retention interval increased, whereas accuracy following the 10 -sec samples declined to well below $50 \%$ correct for all 4 birds. That is, as usual, a clear choose-short bias was observed despite the explicit inclusion of no-sample trials.

An ANOVA performed on the 2-sec versus 10-sec data revealed a main effect of sample duration $[F(1,3)=141.4$, $\left.M S_{\mathrm{e}}=0.004, p<.001\right]$, a main effect of retention interval $\left[F(3,9)=16.40, M S_{\mathrm{e}}=0.007, p<.01\right]$, and a significant interaction $\left[F(3,9)=15.21, M S_{\mathrm{e}}=0.009\right]$. An ANOVA performed on the 2-sec versus no-sample data yielded no significant effects. Once again, as is suggested by the small error bars, power to detect a difference in performance between these two conditions was reasonably high. Using sample standard deviation as an estimate of the true value, power to detect a $10 \%$ difference in performance was .89. However, smaller differences again were less likely to be detected (e.g., power to detect a 5\% difference was .36).

The pattern of results observed in Experiment 2 is consistent with the idea that the birds responded on the basis of the presence versus the absence of the 10 -sec sample. According to this account, the presentation of the choice stimuli served as a cue to retrieve memory for the 10 -sec sample. If no memory of that sample was retrieved (as would typically be true on 2-sec and no-sample trials), the choice alternative not associated with the 10 -sec sample would be selected. If memory of the 10 -sec sample was retrieved (as would typically be true on 10 -secsample trials with a short retention interval), the choice alternative associated with the 10 -sec sample would be selected instead.

In Experiment 1, it appeared that 3 of the 8 birds did not disregard memory for the 2 -sec samples, because their performance on 2-sec-sample trials was associated with a definite forgetting function. Had any of the 4 birds in Experiment 2 been inclined to respond in a similar 


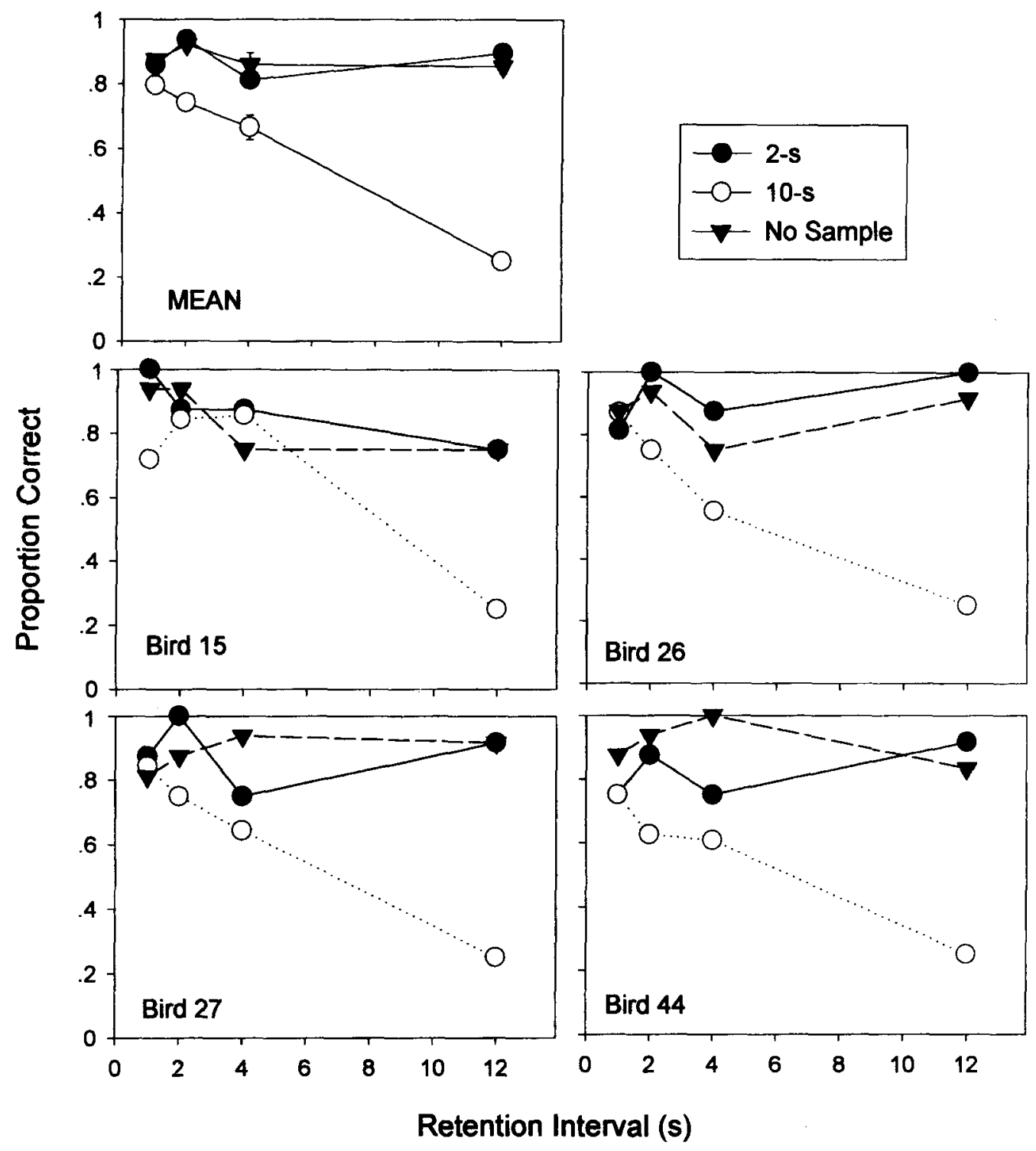

Figure 5. Proportion of correct responses as a function of retention interval on the no-sample, 2-sec-sample, and 10-sec-sample trials (Experiment 2).

fashion, the inclusion of the no-sample trials during training may have induced them to do otherwise. That is, rather than having to learn three rules (namely, if $10 \mathrm{sec}$, choose green; if $2 \mathrm{sec}$, chose red; and if nothing, choose red), these birds could simplify the task by responding on the basis of the presence versus the absence of the 10 sec sample (namely, if $10 \mathrm{sec}$, choose green; otherwise, choose red).

The results of Experiment 2 can also be interpreted in terms of the subjective-shortening model. As in Experiment 1 , the fact that performance on the 2-sec-sample trials was virtually identical to performance on no-sample trials was not necessarily predicted by the subjectiveshortening account, but that account is not contradicted either. For example, it could be that the no-sample trials were construed by the bird as being 0 -sec-sample trials and that the difference between 0 and $2 \mathrm{sec}$ is too small to effect a difference in performance. Alternatively, the procedure may have induced the birds to adopt a detection strategy or a default response strategy that they ordinarily do not rely on when the more standard $2-\mathrm{sec}$ versus $10-$ sec procedure is used.

\section{EXPERIMENT 3}

Experiment 3 was similar to Experiment 2 in every way, except that the correct choice on no-sample trials was now the same as that on 10 -sec-sample trials. Thus, for example, if red was the correct choice following the 2 -sec sample, green would be the correct choice following 
10 -sec samples and on no-sample trials. That being the case, a simple detection strategy the birds could adopt would be to choose red, given a $2-\mathrm{sec}$ sample, and to choose green otherwise. If the birds did adopt that strategy, performances on the 10 -sec-sample trials and the no-sample trials would be equivalent and would be unaffected by the size of the retention interval. If they responded instead on the basis of three decision rules (namely, if $2 \mathrm{sec}$, choose red; if $10 \mathrm{sec}$, choose green; and if no sample, choose green), performance on the 10 -secsample trials would not necessarily be equivalent to performance on the no-sample trials and might exhibit a forgetting function as memory for that sample faded away with increasing retention interval.

Note that if performances on the 10-sec-sample and the no-sample trials do turn out to be equivalent, the usual asymmetry observed in a duration discrimination task will have been reversed. That is, whereas a typical duration discrimination procedure usually yields a chooseshort bias, this one should yield a choose-long bias instead. Thus, for example, if pigeons learn to choose red following a 2-sec sample and to choose green otherwise (i.e., if they rely on the detection strategy one might expect), performance on 2-sec trials should be accurate when the retention interval is short and memory for the 2 -sec sample is strong. However, when the retention interval is long enough, memory of the 2 -sec sample will have faded completely, and given the decision rules it is operating under, the bird should choose green most of the time (i.e., performance on 2-sec-sample trials should drop to below $50 \%$ correct). On both 10 -sec-sample trials and no-sample trials, the bird should choose green most of the time, whether the retention interval is short or long, because in all cases (10-sec sample or no sample, short retention interval or long retention interval), the absence of any memory of the 2-sec sample will lead the bird to choose green most of the time. Hence, a choose-long bias should be observed.

What does the subjective-shortening model predict for this experiment? That depends on what one assumes about the way in which the experimental procedure affects the bird's decision strategy. Assuming that the introduction of no-sample trials does not induce the birds to rely on a default response strategy and assuming that no-sample trials can be thought of as 0 -sec-sample trials, the subjectiveshortening model predicts that performance following the 10 -sec sample will be $\mathrm{U}$-shaped as the retention interval increases (see Kraemer et al., 1985). According to this version of the subjective-shortening account, baseline training in Experiment 3 would teach the bird to choose one choice alternative following 10 -sec and 0 -sec samples and to choose the other alternative following $2-\mathrm{sec}$ samples. When the retention interval is short, a 10 -sec sample will be remembered as such, and the bird will respond correctly. As the retention interval increases, the remembered duration of the $10-\mathrm{sec}$ sample will shrink, so that, at some point, it will be subjectively closer to $2 \mathrm{sec}$. At that point, the pigeon should incorrectly select the choice stimulus as- sociated with the 2 -sec sample. As the retention interval increases still further, the remembered duration of the 10 sec sample may shrink even further, so that, at some point, it will be subjectively closer to $0 \mathrm{sec}$. At that point, the pigeon should once again correctly select the choice alternative associated with both the 10 -sec and the 0 -sec samples. In other words, the forgetting function following the 10 -sec sample ought to be U-shaped. By contrast, performance following the 2-sec sample should be accurate following a short retention interval (because the sample is accurately remembered as having been $2 \mathrm{sec}$ in duration) but should fall below $50 \%$ correct as the retention interval increases. This should happen because the remembered duration of the 2-sec sample will eventually decrease to $0 \mathrm{sec}$, at which point the bird should reliably choose the other (incorrect) choice alternative.

Thus, one version of the subjective-shortening model and the asymmetric salience model both predict a chooselong effect following 2-sec samples in this experiment. The models differ in their predictions about what should happen following the 10 -sec sample. Assuming that the birds rely on analogical coding (which is not a necessary assumption in this case), the subjective-shortening model predicts a U-shaped forgetting function on these trials, whereas the asymmetric salience model predicts a flat forgetting function.

\section{Method}

Subjects. The 4 pigeons used in Experiment 2 were used again in Experiment 3, and they were housed and maintained under the same conditions.

Apparatus. The same experimental apparatus as that used in Experiment 2 was used again here.

Procedure. As was indicated earlier, Experiment 3 was actually run prior to Experiment 2. Before entering this experiment, the birds had been run for many sessions on a standard DMTS task involving differential reinforcement outcomes (food pellets vs. milo), in which the probability of reinforcement was varied across conditions. They had no prior experience on a sample/no-sample procedure or an event duration procedure. Baseline training sessions in this experiment involved 60 trials, consisting of thirty 2 -sec-sample trials, fifteen 10-sec-sample trials, and 15 no-sample (or 0-sec) trials. Table 1 shows the assignment of samples to choice stimuli for each bird. The retention interval was always $1 \mathrm{sec}$, and a correction procedure was in effect. All other procedural details were identical to those of Experiment 2.

After a reasonable performance level had been attained, the correction procedure was discontinued. Once subsequent performance reached stability (defined as five consecutive sessions above $75 \%$ correct overall), probe sessions were introduced. As in Experiment 2 , probe sessions involved retention intervals of $1,2,4$, and $12 \mathrm{sec}$ following each sample. A total of four probe sessions was run for each bird. Each probe session was followed by two sessions of baseline training, in which all retention intervals were again $1 \mathrm{sec}$ long.

\section{Results and Discussion}

Figure 6 presents the main results of Experiment 3. The figure shows the performance of each bird on 2-sec, 10 -sec, and no-sample trials averaged over the four probe sessions, as well as a plot of the data averaged over birds. All 4 birds demonstrated a clear asymmetry between ac- 


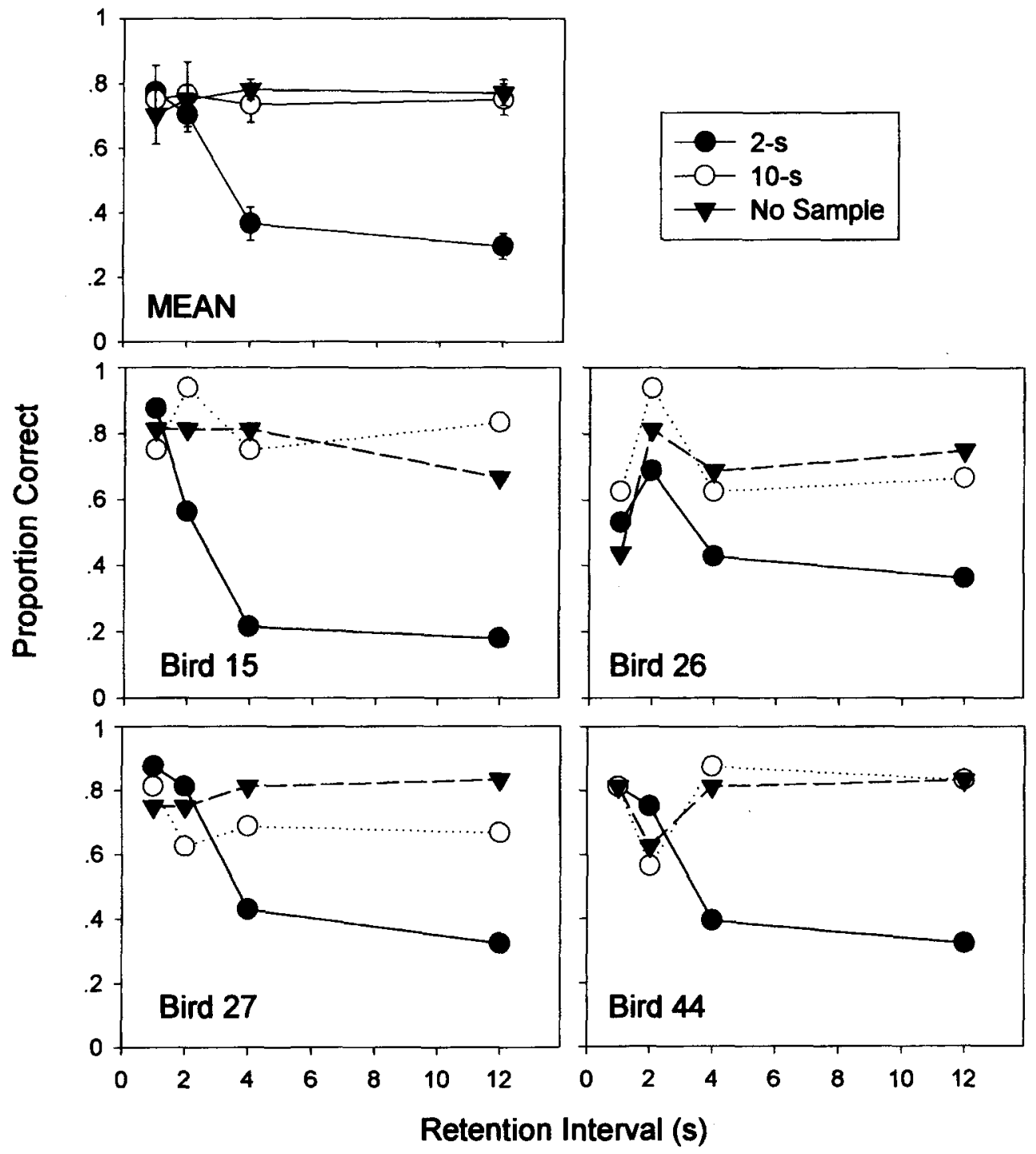

Figure 6. Proportion of correct responses as a function of retention interval on the no-sample, 2-secsample, and 10-sec-sample trials (Experiment 3).

curacy following the 2-sec sample, which decreased to well below $50 \%$ correct as the retention interval increased, and accuracy following the 10-sec and 0-sec samples, which remained fairly constant at well above $50 \%$ correct for all 4 birds. That is, a clear choose-long bias was induced by associating 10-sec samples and no samples with the same choice stimulus. Moreover, the data reveal that the birds responded to the 10 -sec samples and to the no samples in almost exactly the same way (as if they were disregarding memory for the 10 -sec samples altogether). Although 2 birds exhibit slight evidence of a Ushaped retention function following the 10 -sec sample, the other two exhibit slight evidence of an inverted U.

An ANOVA performed on the 2-sec versus 10-sec data revealed a main effect of sample duration $[F(1,3)=$ $\left.14.94, M S_{\mathrm{e}}=0.025, p<.05\right]$, a main effect of retention interval $\left[F(3,9)=8.22, M S_{\mathrm{e}}=0.015, p<.01\right]$, and a significant interaction $\left[F(3,9)=6.52, M S_{\mathrm{e}}=0.016, p<.05\right]$. An ANOVA performed on the 10-sec versus no-sample data yielded no significant effects. Once again, as is suggested by the small error bars, power to detect a difference in performance between these two conditions was reasonably high. Using sample standard deviation as an estimate of the true value, power to detect a $10 \%$ difference in performance was .90 , although power to detect a smaller difference was less (e.g., power to detect a 5\% difference was .37).

The flat forgetting function following the 10 -sec sample is not consistent with one version of the subjectiveshortening model outlined earlier. That account predicts a U-shaped function as the retention interval increases, because the subjective duration of the sample should de- 
crease toward $2 \mathrm{sec}$ at some point and then further decrease toward zero. For all 4 birds, performance was essentially at chance at the longest retention interval, which presumably means that all information about the initiation of the trial was essentially forgotten. Thus, if the subjective duration of the 10 -sec sample did shrink over time, it presumably had shrunk to nearly zero by the end of the 12-sec retention interval. Despite that, none of the 4 birds showed clear evidence of a U-shaped pattern (although 2 showed slight evidence of that pattern).

On the other hand, and as indicated earlier, this could simply mean that the addition of explicit no-sample trials induced the birds to adopt a default response strategy. This strategy would involve choosing the alternative associated with the 10 -sec-sample and no-sample trials by default and choosing the alternative associated with the 2-sec-sample trial only when memory for that sample was present. This, of course, is similar to the detection account we offered above, and, contrary to what a subjectiveshortening account assumes, it is similar to what we argue may be happening in a standard 2-sec versus $10-\mathrm{sec}$ discrimination task as well.

\section{GENERAL DISCUSSION}

The results of all three experiments are consistent with the idea that pigeons can transform a nominal discrimination task into a detection task. More specifically, the results suggest that the birds can respond on the basis of the presence versus the absence of one of the samples, effectively disregarding any memory of the other sample that might otherwise be available. Experiment 1 showed that the performance of the 5 birds exhibiting evidence of a choose-short effect was unaffected when the 2-secsample trials were replaced by no-sample trials for five sessions. This is as it should be if the presentation of the choice stimuli occasions a search of memory for the 10-sec sample (but not of memory for the 2-sec sample). Eliminating disregarded samples simply arranges procedurally what was already happening psychologically.

Experiment 2 showed that when explicitly programmed no-sample trials were associated with the same choice alternative as the 2-sec samples, birds again responded identically on the 2-sec- and no-sample trials (at least within the limits of measurement error). Performance on these trials was unaffected by the size of the retention interval, whereas performance on 10-sec-sample trials decreased rapidly to well below $50 \%$ correct. As was the case with Experiment 1, this result is consistent with the idea that the birds learned to solve this task on the basis of the presence or absence of memory for the 10-sec sample.

Given that 2-sec samples and no samples were both associated with the same choice alternative in Experiment 2, it makes sense that the birds learned to respond on the basis of the presence or absence of the 10-sec sample. Why, though, would they also adopt that strategy on the standard 2-sec versus $10-\mathrm{sec}$ procedure, which does not involve no-sample trials? The answer is not clear, but perhaps the birds are inclined to transform a discrimination task into a detection task simply because they find it easier to selectively focus attention on the more salient of two events.

Experiment 3 showed that when explicitly programmed no-sample trials were associated with the same choice alternative as the 10 -sec samples, the birds appeared to reverse the strategy described above by responding on the basis of the presence or absence of memory for the 2-sec sample. Thus, accuracy on both 10 -sec and no-sample trials remained constant (and equivalent) as the retention interval increased, whereas performance on the 2-secsample trials decreased rapidly to less than $50 \%$ correct. This result indicates that pigeons may be quite flexible about which memory they selectively search for when presented with choice stimuli. Although they may be naturally inclined to search for memory of the more salient sample, they can, according to this account, be induced to search for memory of the less salient sample if it is efficient to do so.

Related to this, Grant and Kelly (1996) showed that a choose-short effect can be obtained even when the two samples differ only slightly in terms of salience. They used sample durations of 2 versus $3 \mathrm{sec}$ in their experiment. Although the task proved to be difficult for the birds to learn (requiring thousands of training trials and the use of a correction procedure at all times), they did eventually acquire it, and they did show evidence of a choose-short effect. Thus, from our point of view, this suggests that birds can rely even on slight differences in salience in order to respond on the basis of the presence or absence of the more salient sample. It is apparently not critical that the stimuli in question differ greatly in magnitude but only that they do differ.

All of these results suggest a simple principle that may apply generally to pigeon memory: Whenever the sample stimuli consist of two points along a prothetic, or quantitative, continuum (Stevens, 1957), pigeons may be inclined to solve the task on the basis of the presence versus the absence of one stimulus level. By contrast, pigeons may be less likely to rely on a detection strategy when the stimuli are drawn from a metathetic, or qualitative, continuum (e.g., color or pitch). For prothetic stimuli, birds may be naturally inclined to focus on the stimulus that is higher in magnitude (even if it is not much higher in magnitude), but the results of Experiment 3 show that they can focus on the lower magnitude stimulus if it is more efficient to do so.

\section{Previous Accounts of the Role of Nothing}

Several previous accounts assume that the chooseshort phenomenon reflects the fact that "nothing" is more similar to a 2 -sec event than to a $10-\mathrm{sec}$ event. Thus, when memories fade to nothing following a long retention interval, pigeons select the 2 -sec choice alternative because it is the best of the two available options (Killeen \& Fetterman, 1988; Kraemer et al., 1985; Sherburne, Zentall, \& Kaiser, 1998). The results of Experiments 1 and 2 
are consistent with this idea, except that the identical performances on the 2-sec and no-sample trials would not necessarily have been predicted. The results of Experiment 3 , however, which revealed equivalent performances on 10-sec and no-sample trials, suggest that mere similarity between $2 \mathrm{sec}$ and nothing may not be all that is involved. Rather, the critical point may be that the sample stimuli are derived from a prothetic continuum and that, under those conditions, birds can respond on the basis of the presence or absence of either the higher or lower magnitude stimulus (although they may be naturally inclined to rely on the stimulus of higher magnitude).

\section{The Default Response Model}

To this point, we have alluded to the fact that the detection account we propose is similar to the default response model that has been advanced to explain the asymmetric forgetting functions observed on sample/no-sample tasks (e.g., Grant, 1991). Although the default response account is very similar to the detection account, they are different (see Wixted, 1993), and those differences are theoretically important. The difference between the default response account and the detection account is most easily illustrated by considering some compelling evidence against the default response interpretation of the chooseshort effect, reported by Grant and Spetch (1994). They trained birds on a 2 -sec versus 10 -sec task and on a 4.5$\mathrm{sec}$ versus $22.5-\mathrm{sec}$ task simultaneously. The $2-\mathrm{sec}$ and 10 -sec samples were associated with one set of choice stimuli (e.g., red and green), and the 4.5-sec and 22-sec samples were associated with another set (e.g., vertical and horizontal lines). A choose-short effect was evident in both cases. On transfer trials, the short samples were followed by the two choice stimuli that had been associated with the 2-sec and 4.5-sec samples (e.g., red and vertical lines). If pigeons learned to solve both tasks by choosing red and vertical lines by default, performance on these transfer trials should be at chance (because the bird would be confused about which one to choose by default). Contrary to this prediction, performance was better than chance, which indicates that memory for the short stimuli must have been accessed.

These findings are inconsistent with the default response account, but they are not incompatible with the detection account offered here. According to the detection account, the presentation of the choice stimuli cues the bird to search for evidence that the long-duration sample occurred at the beginning of the trial. Thus, for example, if 2-sec and 10-sec samples were associated with red and green choice stimuli, respectively, the appearance of red and green would cue the bird to search for memory of the 10-sec sample. An important and unique assumption of this model is that a retrospective memory search occurs on every trial (and this is the critical difference between the detection account and the default response model). If evidence for the 10-sec sample exceeds a criterion, one choice alternative is selected; otherwise, the other choice is made. This is not to say that the bird has no memory of the 2-sec sample or that it has forgotten which choice alternative is associated with that sample (which the bird presumably learned during training). The model simply assumes that, at some point, the bird adopts a strategy such that memory for the 2-sec sample is not typically accessed and, therefore, ordinarily plays no role.

When faced with a novel arrangement of choice stimuli (red and vertical lines), as in the study by Grant and Spetch (1994), the cue that theoretically occasions a search of memory for the $10-\mathrm{sec}$ sample is not present. Under these conditions, the birds may instead respond on the basis of their memory for the 2 -sec or the $4.5-\mathrm{sec}$ samples, because the only retrieval cues available are the ones associated with those samples. Successful retrieval of those memories would facilitate the above-chance performance that Grant and Spetch observed on these probe trials. Chance performance is only predicted by a theory that assumes that pigeons execute a default choice response when memory for the $10-\mathrm{sec}$ sample is not present to override that strategy.

\section{General Applicability of the Detection Model}

One strength of the subjective-shortening model is its ability to explain a wide array of findings from the duration discrimination literature above and beyond the choose-short effect. For example, Spetch (1987) showed that if a long retention interval (e.g., $10 \mathrm{sec}$ ) is exclusively used during baseline training in a duration discrimination task, the introduction of shorter retention intervals in probe sessions results in a choose-long effect. That is, on 2-sec-sample trials, pigeons are inclined to choose the 10sec choice alternative following an unexpectedly short retention interval.

Intuitively, this seems like an odd result, because memory for the 2-sec sample should be quite strong following a short retention interval, and the birds have presumably learned during the course of training which choice alternative to select on 2-sec-sample trials. Thus, if anything, it seems that they should be more inclined to choose the 2 -sec choice alternative when the retention interval is short. Instead, they are more inclined to choose the 10 $\mathrm{sec}$ alternative. Although this result is counterintuitive, it is exactly what should be observed according to the subjective-shortening account. The reason is that, following a 10-sec retention interval (the interval used during training), the subjective durations of the 10-sec and 2-sec samples will both have decreased to smaller values. After $10 \mathrm{sec}$ of delay, the 10-sec sample may, for example, be subjectively represented by a value closer to $2 \mathrm{sec}$, and the 2 -sec sample may be subjectively represented by a value closer to, say, $0.5 \mathrm{sec}$. Under such conditions, the introduction of a short retention interval following a 2-sec sample should induce the bird to choose the wrong alternative (because the 2-sec sample will be subjectively represented as something close to $2 \mathrm{sec}$ ).

It may be possible to accommodate these results by the detection model outlined earlier (Wixted \& Dougherty, 1996), but it seems fair to say that this choose-long effect 
is not obviously predicted by the model. Another finding that follows naturally from the subjective-shortening account (but not from the detection account) was reported by Spetch and Wilkie (1983). They trained 2 pigeons to discriminate three durations (short, medium, and long), using a three-key choice procedure. When retention intervals were introduced, a choose-short effect was observed following medium-duration samples, as the subjectiveshortening model predicts. Following long-duration samples, pigeons at first exhibited a tendency to make incorrect choices to the medium comparison as the retention interval increased. With further increases in the retention interval, a tendency to make incorrect choices to the short comparison was observed. This is exactly what should happen if the representation of the long sample decreased in a continuous way as the retention interval increased. Although this result is compatible with the subjective-shortening model, it is not directly predicted by the idea that the pigeons adopted a detection strategy when exposed to the three-key task.

In light of these considerations, it should be clear that our point is not that the subjective-shortening account is incorrect or that all of the data ordinarily explained by that model can be explained by the detection account. Rather, our point is that, under some circumstances, pigeons seem to be able to transform a nominal discrimination task into a detection task. If so, findings that are ordinarily assumed to reflect a shrinking memory representation (namely, the choose-short effect) may sometimes arise for an altogether different reason.

\section{REFERENCES}

ColwILl, R. M. (1984). Disruption of short-term memory for reinforcement by ambient illumination. Quarterly Journal of Experimental Psychology, 36B, 235-258.

Fetterman, J. G. (1995). The psychophysics of remembered duration. Animal Learning \& Behavior, 23, 49-62.

FetTerman, J. G., \& MacEwen, D. (1989). Short-term memory for responses: The "choose-small" effect. Journal of the Experimental Analysis of Behavior, 52, 311-324.

Grant, D. S. (1991). Symmetrical and asymmetrical coding of food and no-food samples in delayed matching in pigeons. Journal of $E x$ perimental Psychology: Animal Behavior Processes, 17, 186-193.

Grant, D. S., \& Kelly, R. (1996). The role of minimum wait time and sample discriminability in the coding of event duration in pigeons. Learning \& Motivation, 27, 243-259.

Grant, D. S., \& Kelly, R. (1998). The effect of variable-delay training on coding of event duration in pigeons. Learning \& Motivation, 29, 49-67.

Grant, D. S., \& SPETCH, M. L. (1994). The role of asymmetrical coding of duration samples in producing the choose-short effect in pigeons. Learning \& Motivation, 25, 413-430.

Killeen, P. R., \& Fetterman, J. G. (1988). A behavioral theory of timing. Psychological Review, 95, 274-295.

Kraemer, P. J., Mazmanian, D. S., \& Roberts, W. A. (1985). The choose-short effect in pigeon memory for stimulus duration: Subjective shortening versus coding models. Animal Learning \& Behavior, 13, 349-354.

Sherburne, L. M., \& Zentall, T. R. (1993). Coding of feature and no-feature events by pigeons performing a delayed conditional discrimination. Animal Learning \& Behavior, 21, 92-100.

Sherburne, L. M., Zentall, T. R., \& Kaiser, D. H. (1998). Timing in pigeons: The choose-short effect may result from pigeons' "confusion" between delay and intertrial intervals. Psychonomic Bulletin \& Review, 5, 516-522.

SPETCH, M. L. (1987). Systematic errors in pigeons' memory for event duration: Interaction between training and test delay. Animal Learning \& Behavior, 15, 1-5.

SPETCH, M. L., \& WILKIE, D. M. (1982). A systematic bias in pigeons' memory for food and light durations. Behaviour Analysis Letters, 2 , 267-274.

SperCH, M. L., \& WILkIE, D. M. (1983). Subjective shortening: A model of pigeons' memory for event duration. Journal of Experimental Psychology: Animal Behavior Processes, 9, 14-30.

STEvens, S. S. (1957). On the psychophysical law. Psychological Review, 64, 153-181.

WILSON, B., \& BOAKES, R. A. (1985). A comparison of the short-term memory performances of pigeons and jackdaws. Animal Learning \& Behavior, 13, 285-290.

WIXTED, J. T. (1993). A signal detection analysis of memory for nonoccurrence in pigeons. Journal of Experimental Psychology: Animal Behavior Processes, 19, 400-411.

WIXTED, J. T., \& DOUGHERTY, D. H. (1996). Memory for asymmetric events. In D. L. Medin (Ed.), The psychology of learning and motivation (Vol. 35, pp. 89-126). San Diego: Academic Press.

(Manuscript received June 28, 1999; revision accepted for publication November 12, 1999.) 